\title{
A Remark on Rational Isochronous Potentials
}

Oleg A CHALYKH ${ }^{\dagger}$ and Alexander P VESELOV ${ }^{\ddagger}$

$\dagger$ Department of Applied Mathematics, University of Leeds

Leeds, LS2 9JT, UK

E-mail: o.chalykh@mail.ru

$\ddagger$ Department of Mathematical Sciences, Loughborough University

Loughborough, LE11 3TU, UK

and Landau Institute for Theoretical Physics, Kosygina 2, Moscow, 117940, Russia

E-mail: A.P.Veselov@lboro.ac.uk

This article is part of the special issue published in honour of Francesco Calogero on the occasion of his 70th birthday

\begin{abstract}
We consider the rational potentials of the one-dimensional mechanical systems, which have a family of periodic solutions with the same period (isochronous potentials). We prove that up to a shift and adding a constant all such potentials have the form $U(x)=\frac{1}{2} \omega^{2} x^{2}$ or $U(x)=\frac{1}{8} \omega^{2} x^{2}+c^{2} x^{-2}$.
\end{abstract}

\section{Introduction}

Consider one-dimensional mechanical system with the Hamiltonian

$$
H=\frac{1}{2} p^{2}+U(q)
$$

and ask when such a system has a family of oscillatory solutions with the same period $T$. In that case we will call the corresponding potential isochronous. This is a very old question going back to Huygens and discussed in the classical textbooks on mechanics (see Appell [1], section 213 and Landau-Lifschitz [2], section 12) and in several papers of which we mention [3], [4].

The aim of this note is to point out that although the space of the isochronous potentials is pretty large (see the general description in the next section) only few of them are rational functions. Our main result is the following

Theorem 1. Up to a shift $x \rightarrow x+a$ and adding a constant all rational isochronous potentials have the form $U(x)=\frac{1}{2} \omega^{2} x^{2}$ or

$$
U(x)=\frac{1}{8} \omega^{2} x^{2}+\frac{c^{2}}{x^{2}},
$$

where $c$ is any non-zero constant and $\omega=2 \pi / T$. 
We had not found this result in the literature although some considerations of [4] are very close to it. The proof is actually not difficult anyway (see next section). The reason we find this result important is that it demonstrates an exceptional nature of the CalogeroMoser system [5]. This system describes the pairwise interaction of the particles on the line with the potential

$$
V\left(x_{1}, \ldots, x_{n}\right)=\frac{1}{2} \omega^{2} \sum x_{i}^{2}+C \sum\left(x_{i}-x_{j}\right)^{-2} .
$$

It is known [6] that the motion of this system is periodic with the same period $T=\pi / \omega$. In two-particle case this reduces to the isochronicity of the potential (1.1).

The isochronicity of the family (1.1) can be proved in many ways, of which the most simple one is probably the following. Consider two-dimensional isotropic oscillator with the Hamiltonian

$$
H=\frac{1}{2}\left(p_{1}^{2}+p_{2}^{2}\right)+\frac{1}{2} \omega^{2}\left(q_{1}^{2}+q_{2}^{2}\right) .
$$

The dynamics of the distance $r=\left(q_{1}^{2}+q_{2}^{2}\right)^{1 / 2}$ is described by the one-dimensional system with effective potential $U(r)=\frac{1}{2} \omega^{2} r^{2}+C r^{-2}$, where constant $C$ is half of the square of the angular momentum. Now since the motion of the isotropic harmonic oscillator is periodic with the period $T=2 \pi / \omega$ and the orbits are ellipses the motion in the potential $U(r)$ is also periodic with the period $T=\pi / \omega$ (coefficient $1 / 2$ is due to the central symmetry of the ellipses).

We do not know much about multidimensional generalisations of our result. Some interesting examples can be found in the recent paper [7] by Calogero, who did a lot to revitalise this area. Our interest to this problem was to a large extent stimulated by his enthusiasm.

\section{Proof of the Theorem}

Let us first note that isochronous motion is possible only in the interval where the potential $U(x)$ has the only (and thus global) minimum. Indeed the presence of any other extrema will lead to the divergence of the period given by the integral

$$
T=\sqrt{2} \int \frac{d x}{\sqrt{E-U(x)}}
$$

(see [2]), which must be independent on the energy $E$ (by analyticity arguments).

Let us assume for convenience that the period of oscillations $T=\sqrt{2} \pi$ (so that $\omega=\sqrt{2}$ and the corresponding harmonic potential is simply $U=x^{2}$ ) and that potential energy of the equilibrium is zero. This means that our rational function $y=U(x)$ has a local minimum $x=x_{0}$ with $y_{0}=U\left(x_{0}\right)=0$.

Let $x_{1}(y)$ and $x_{2}(y)$ be corresponding local solutions of the equation $U(x)=y$, i.e. the branches of the inverse function $U^{-1}(y)$ bifurcating from $x_{0}: x_{1}(0)=x_{2}(0)=x_{0}, x_{1}(y) \leq$ $x_{2}(y)$ for positive $y$.

The classical criterium of isochronicity is that the difference

$$
\Delta(y)=x_{2}(y)-x_{1}(y)
$$


is exactly the same as for the corresponding harmonic oscillator (see [2], section 12). In particular for our choice of $T$ we must have

$$
x_{2}(y)-x_{1}(y)=2 \sqrt{y}
$$

Thus if $y=U(x)$ is such a function then $y=U(x)$ implies $y=U(x+2 \sqrt{y})$ for all $(x, y)$ in some open domain $\Omega \subseteq \mathbb{R} \times \mathbb{R}_{+}$. Let us start by considering a more general situation when $y=U(x)$ is a branch of an algebraic function, so that the graph $y=U(x)$ belongs to an irreducible curve $F(x, y)=0$. Making substitution $y=z^{2}$ we get a (possibly reducible) curve $G(x, z)=F\left(x, z^{2}\right)=0$. Then the isochronicity means that two complex algebraic curves $\Gamma: G(x, z)=0$ and $\Gamma^{\prime}: G(x+2 z, z)=0$ have a one-dimensional component in common. If $\Gamma$ and, hence, $\Gamma^{\prime}$ were irreducible, we would get $\Gamma=\Gamma^{\prime}$, so it would mean that for any $(x, z) \in \Gamma,(x+2 n z, z) \in \Gamma$ for any $n \geq 1$. This would mean that $G(x, z)$ does not depend on $x$, which is not interesting.

Thus, $\Gamma$ must be reducible. Now, the only option is that $G(x, z)=P(x, z) P(x,-z)$, where $P$ is irreducible polynomial. Indeed since $G(x, z)$ is invariant under the involution $\tau:(x, z) \mapsto(x,-z)$, the irreducible factors must be interchanged by $\tau$. We can have only one orbit, otherwise the product of the factors along the orbits would give a factorization of $F$.

Since $G=G(x, z)$ and $G^{\prime}=G(x+2 z, z)$ must have a common divisor, we have four cases to consider: either $P(x, z)=c P(x+2 z, z)$ or $P(x, z)=c P(x+2 z,-z)$, or $P(x,-z)=c P(x+2 z, z)$, or, finally, $P(x,-z)=c P(x+2 z,-z)$ for some constant $c$.

The first and the last cases are impossible unless $P$ does not depend on $x$. In the second case, after passing to $u=x+z$ and $v=z$, we get that the (still irreducible) polynomial $Q(u, v):=P(u-v, v)$ has a symmetry $Q(u, v)=c Q(u,-v)$. Thus, either $c=-1$ and $Q$ is odd in $v$, or $c=1$ and $Q$ is even in $v$. If $Q$ is odd in $v$, it is reducible unless $Q=v$. In that case $P=z$ which is not interesting. Thus, the only option is that $c=1$ and $Q$ is any irreducible polynomial that is even in $v$-variable. For example, taking $Q=v^{2}+u$ gives $P=z^{2}+x+z, G(x, z)=\left(z^{2}+x+z\right)\left(z^{2}+x-z\right)$, and $F(x, y)=(y+x+\sqrt{y})(y+x-\sqrt{y})=(y+x)^{2}-y=0$ defines an isochronous potential $y=u(x)$.

The harmonic oscillator corresponds to $Q=u$ which gives $P=x+z, G(x, z)=(x+$ $z)(x-z)$, so $F(x, y)=x^{2}-y$. Similarly, in the third case, when $P(x, z)=c P(x-2 z,-z)$, we pass to $u=x-z$ and $v=z$ to get $Q(u, v):=P(u+v, v)=c P(u-v,-v)=c Q(u,-v)$, so as above we get that $c=1$ and the irreducible polynomial $Q$ is even in $v$-variable.

Now, returning to our original question when $U(x)$ is rational, we have $F(x, y)=$ $a(x) y+b(x)$ for some polynomials $a, b$. Then the reducibility of $G(x, z)=a z^{2}+b$ gives that the factors must be linear in $z$, so up to a constant factor, $a z^{2}+b=(\alpha+\beta z)(\alpha-\beta z)$ where $\alpha, \beta$ are some polynomials in $x$. As we saw above, we must have that either $\alpha(x+$ $2 z)-\beta(x+2 z) z \equiv \alpha(x)+\beta(x) z$ or $\alpha(x-2 z)-\beta(x-2 z) z \equiv \alpha(x)+\beta(x) z$, identically in $x, z$. Note that the second case reduces to the first by changing $\beta$ to $-\beta$ and $z$ to $-z$. Thus, we have only to consider the first functional equation:

$$
\alpha(x+2 z)-\beta(x+2 z) z \equiv \alpha(x)+\beta(x) z .
$$

In that case, first differentiate with respect to $z$ at $z=0$, this gives $\alpha^{\prime}(x)=\beta(x)$. Next, put $x=0, z=t / 2$, this gives:

$$
\alpha(t)=\alpha(0)+t / 2(\beta(t)+\beta(0)) .
$$


Now, differentiating this with respect to $t$ and using the previous relation, we get: $t \beta^{\prime}(t)=$ $\beta(t)-\beta(0)$, which gives that $\beta$ is linear polynomial, $\beta(x)=C x+C_{1}$. By integrating, we find that $\alpha(x)=1 / 2 C x^{2}+C_{1} x+C_{0}$. Now it is easy to check directly that for such $\alpha, \beta$ the functional equation is satisfied.

This gives us that $G(x, z)=\alpha^{2}-\beta^{2} z^{2}$ and $u(x)=\alpha^{2} / \beta^{2}$. We have two cases: $C=0$, in which $U(x)=x^{2}$ up to a shift in $x$, and the case $C \neq 0$, in which we may assume that $C=1$ and find that up to a shift in $x, U(x)=1 / 4 x^{2}+g^{2} x^{-2}-g$ with $g=1 / 2 C_{1}^{2}-C_{0}$. Note that $g=0$ is not allowed since in that case $P(x, z)$ and $F(x, y)$ are reducible. This completes the proof of the Theorem.

\section{Discussion: quantum analogs.}

A natural quantum analog of our problem is to describe the one-dimensional Schrödinger operators

$$
L=-\frac{d^{2}}{d x^{2}}+u(x)
$$

with the equidistant spectrum, i.e. isospectral to the usual harmonic oscillator. The last problem was investigated by McKean and Trubowitz [8] and Levitan [9]. The relation between isospectrality and isochronicity was discussed by Eleonski et al in [10]. In these papers the potential $U(x)$ was assumed to be regular on the whole $x$-axis.

On the other hand there is "dressing chain" approach [11], which allows to construct a wide class of the operators with the spectrum consisting of several arithmetic progressions. The corresponding potentials satisfy Painlevé-type equations and do not belong to the functional class considered in $[8,9]$ even in the case when they are regular on the whole axis. But in general the potentials produced by this approach have poles on the real axis which makes the investigation of the spectral problem less straightforward.

An interesting question therefore is to describe all singular rational potentials with equidistant spectrum on one of the (possible two) semi-lines. We conjecture that the answer is the same as in the isochronicity problem, i.e. up to a shift such potentials have the form

$$
u(x)=A x^{2}+\frac{B}{x^{2}} .
$$

The equidistance property of the corresponding spectra is well-known in quantum mechanics (see e.g. Landau-Lifschitz [12], section 36). An interesting novelty of the quantum case is that here parameter $B$ can be negative (but larger than $-\frac{1}{4}$ ).

\section{Acknowledgements}

We are grateful to Holger Dullin and Sasha Pushnitski for useful discussions.

\section{References}

[1] Appell P. Traite de mechanique rationelle, tome 1. Gauthieres-Villars, Paris, 1902.

[2] Landau L.D. and Lifschitz E.M. Mechanics. Pergamon, London, 1981. 
[3] Urabe M. Potential forces which yield periodic motions of a fixed period. J. Math. Mech. 10 (1961), 569-578.

[4] Cima A., Manosas F., and Villadelprat J. Isochronicity for several classes of Hamiltonian systems. J. Diff. Eq. 157 (1999), 373-413.

[5] Calogero F. Solution of the one-dimensional N-body problems with quadratic and/or inversely quadratic pair potentials. J. Math. Phys. 12 (1971), 419-436.

[6] Olshanetsky M.A., Perelomov A.M. Geodesic flows on symmetric spaces of zero curvature, and explicit solutions of the generalized Calogero model for the classical case. Funkt. Anal. Appl. 10 (1976), no. 3, 86-87.

[7] Calogero F. Two new classes of isochronous Hamiltonian systems. J. Nonlinear Math. Phys. 11 (2004), no. 2, 208-222.

[8] McKean H. P. and Trubowitz E. The spectral class of the quantum-mechanical harmonic oscillator. Comm. Math. Phys. 82 (1981/82), no. 4, 471-495.

[9] Levitan B. M. Sturm-Liouville operators on the entire real axis with the same discrete spectrum. Math. USSR-Sb. 60 (1988), no. 1, 77-106.

[10] Eleonski V.M., Korolev V.G. and Kulagin N.E. On a classical analog of the isospectral Schrödinger problem. JETP Letters, 65 (1997), no.11, 889-893.

[11] Veselov A.P. and Shabat A.B. Dressing chain and spectral theory of Schrödinger operators. Funct. Anal. Appl., 27(2) (1993), 81-96.

[12] Landau L.D. and Lifschitz E.M. Quantum Mechanics: Non-relativistic Theory. Pergamon, Oxford, 1977. 